\title{
A Generic Standalone Design Approach to Embedded Hardware Development
}

\author{
Pranav Jayaram ${ }^{1}$, Rakesh B V $\mathrm{V}^{2}$, Pooja $\mathrm{N}^{3}$, Madhurya $\mathrm{R}^{4}$ and Swamy $\mathrm{TN}^{5}$ \\ UG students ${ }^{1}$ and Assistant Professor ${ }^{5}$ \\ ${ }^{1-5}$ Department of Electronics and Communication Engineering \\ Dr. Ambedkar Institute of Technology \\ Bengaluru, India \\ India
}

\begin{abstract}
Hardware design in an Electronic System refers to a holistic design incorporating the basic schematic, the board layout, routing, fabrication, and testing. Present industry trends use virtual design methodologies to construct one or more printed circuit boards $(P C B$ 's) which implement interconnections between components and are manufactured in bulk for multiple applications in Consumer Electronics, Automobiles, Computers and Entertainment Systems. They even find application is a diverse spectrum of micro to mega-scale of electronic products.

This paper presents a novel approach to design a proprietary standalone controller card. Hardware Design integrates custom Schematic, Board layout, and manual track routing. The Standalone design technique provides a technique to physically connect microcontrollers to a master device for the sole purpose of firmware upload. The Two fold approach refers to a standard OEP (Original Entry Point) based design juxtaposed with an optional DE (Dual Entry Design) for an additional Proprietary entry point included to serve as an irretrievable secure secondary dumping mechanism. This offers flexibility to upload firmware securely as an extension to the prevalent OEP technique. The final design integrates the PCB's functionality of interconnected components with accurate placement and feasible dimensions for large-scale production.
\end{abstract}

Key Words: PCB, OEP, Standalone, Embedded, Proprietary, Hardware.

\section{INTRODUCTION}

The first step of Hardware design is to assert the need for a generic design, a schematic, Bill of Materials, Component Placement, Routing Considerations, and Layout Visualization. Upon successful completion of the basic design technique, the postfabrication steps are taken to ensure the testing and working of the fabricated Hardware[2].

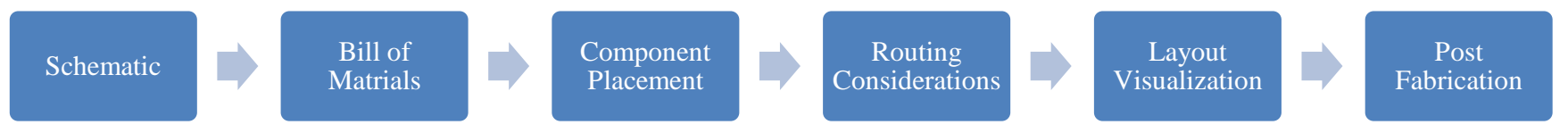

Figure 1 Design Technique 
International Journal of Advances in Scientific Research and Engineering (ijasre), Vol 5 (6), June-2019

\section{GENERIC CONTROLLER CARD DESIGN (TYPE A: OEP BASED DESIGN)}

A complete design methodology of a customizable controller card is illustrated below. This design illustrates an Original Entry Point (OEP) based approach. This process entails design of the schematic which conforms to original design rules that are rigid in nature and typically use a standard in-built USB or S/W technique of code dumping. The Schematic of the Controller card is highly simplistic in nature with an option to expand the design with a port-based approach of peripheral connectivity. Nevertheless, the schematic requires an Error Rule Check (ERC) which is used to minimize errors during design. The design Bill of Materials is also reduced with the use of a generic controller. The Component placement is slightly complex due to the legacy considerations of the controller design. The routing Considerations are high with definitive Design Rule Checks (DRC). The Layout can be visualized with the use of 3D modeling API's and the Post Fabrication Testing of the Design is significantly simple.

Table 1 Design Considerations and Complexity of Original Entry Point based Hardware Design

\begin{tabular}{|l|l|}
\hline DESIGN CONSIDERATION & COMPLEXITY \\
\hline Schematic Design & Simple \\
\hline Bill of Materials & Reduced \\
\hline Component Placement & Complex \\
\hline Routing Considerations & High \\
\hline Layout Visualization & Simple \\
\hline Post Fabrication/Testing & Effective \\
\hline
\end{tabular}

\subsection{Schematic Design (OEP Design)}

The Original Entry point-based design uses an existing design for a controller schematic which additional peripherals and routing considerations. As seen in Figure 2, the schematic uses a standard design of an ATMega328 controller card from the Application note of the manufacturer. Other components illustrated depict the peripherals used[1].

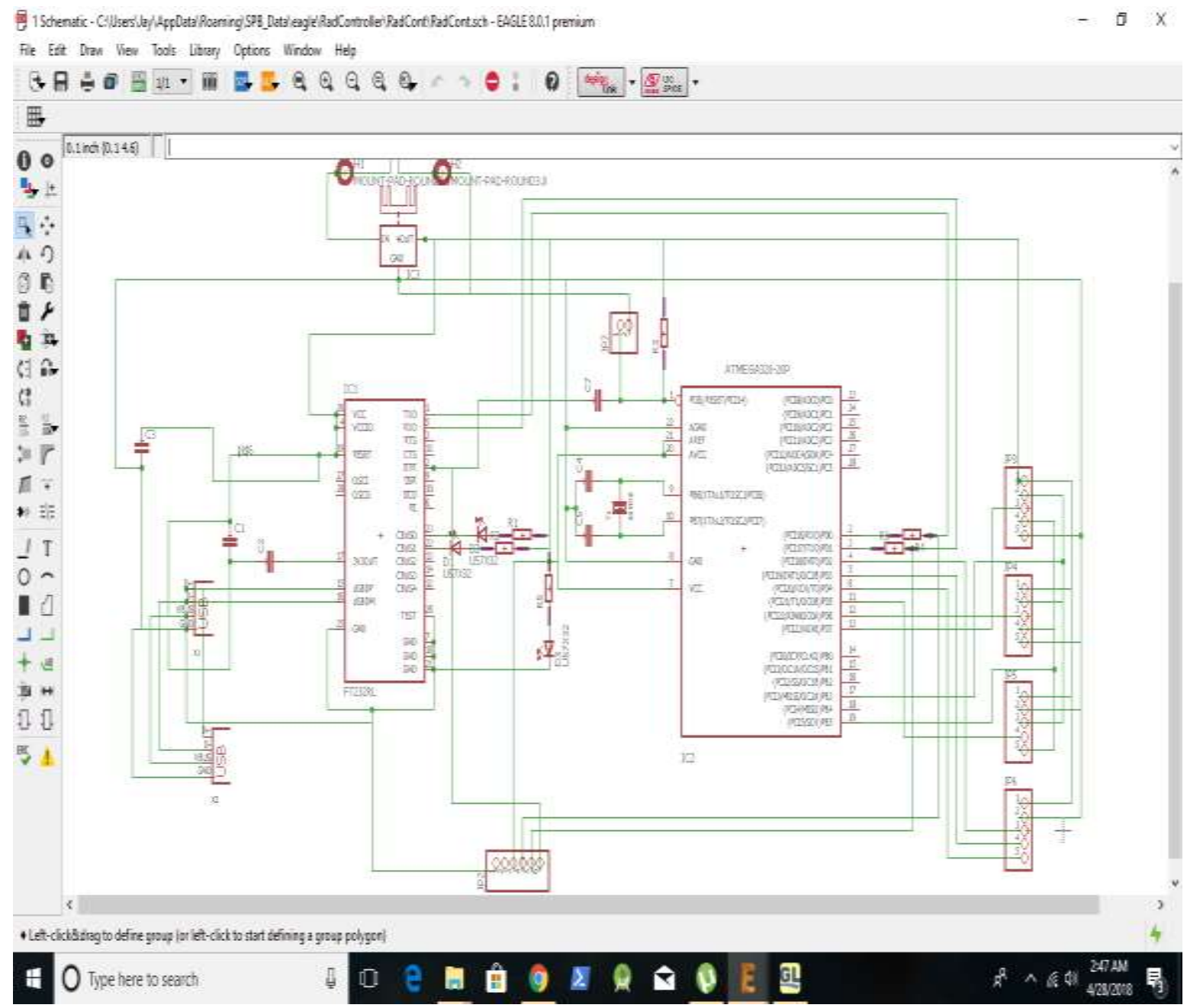

Figure 2 OEP Schematic Designed using Eagle 


\subsection{Component Placement (OEP Design)}

The component Placement defined in Figure 3 incorporates ports for peripherals such as Power Ports, Wireless Connectivity (Bluetooth), Reset Port, Digital and Analog O/P ports.

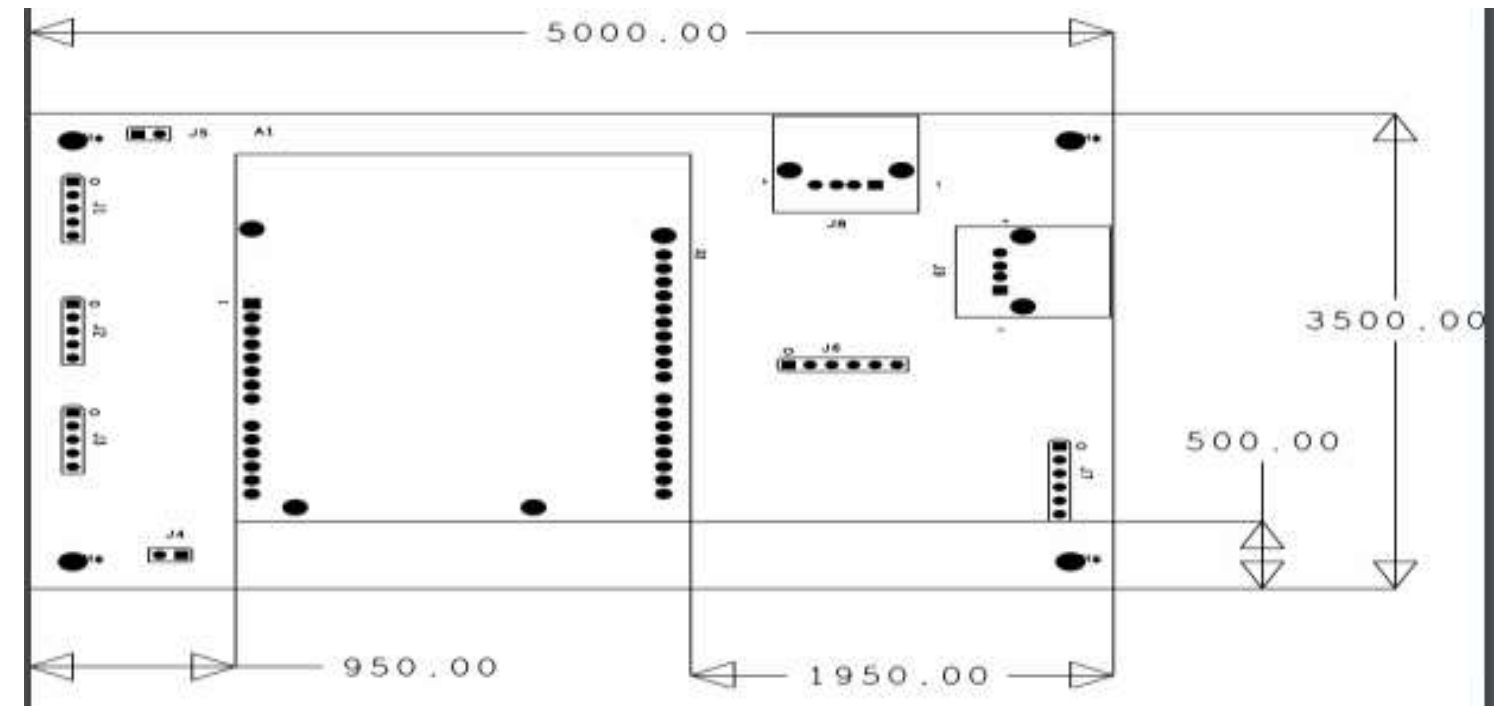

Figure 3 Component Placement of OEP Design

\subsection{Board Layout (OEP Design)}

The Board Layout of the OEP has the complete routing and track considerations of all layers of the PCB designed. The Power supply and Ground tracks use a $0.4 \mathrm{~mm}$ track width for undeterred power transmission with EMI Shielding and reduced Power losses. The Digital signals are designed with a standard track width of $0.24 \mathrm{~mm}$. Excess track width in digital signals induces an unwanted capacitive charge which later induces latency in signal transmission. The tracks designed provide a standard convention of further designs illustrated in this paper[3].

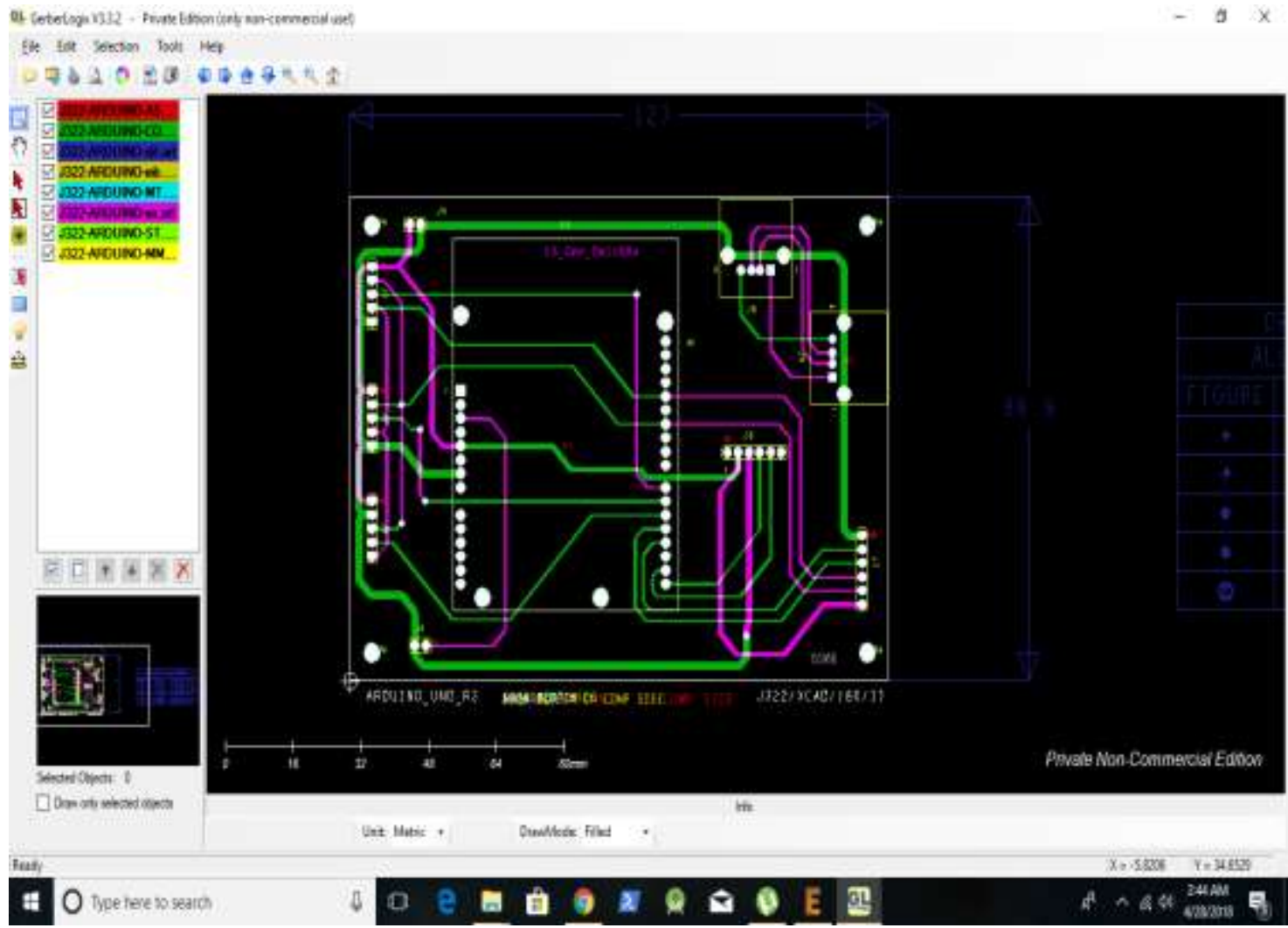

Figure 4 Board Layout for OEP Design 


\subsection{Dimension for Fabrication (OEP Design)}

As seen in Figure 5, the dimensions (in $\mathrm{mm}$ ) of the PCB are mentions for Plated and non-plated areas of the PCB. The CNC Fabrication requires a complete dimension matrix in a vector file for fabrication[2].

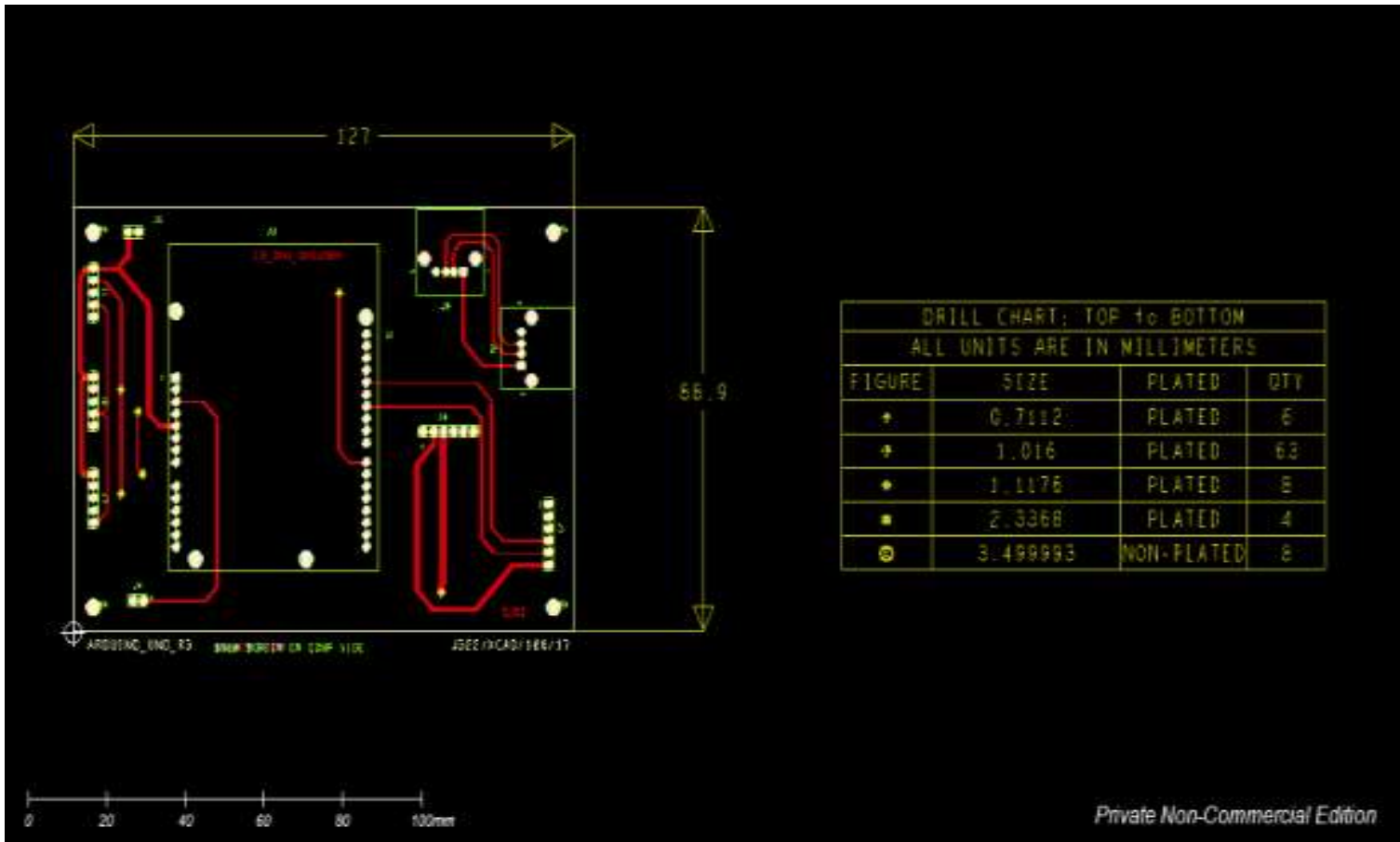

Figure 5 Dimensions in mm for Fabrication

\subsection{Post Fabrication and Design Verification (OEP Design)}

The Original Entry Point based design uses a standard assembly based verification technique. Here, the complete component assembly is done and tracks are checked with the help of a Multimeter and in some rare cases, FPT (Flying Probe Testing) can also be done in order to verify the design. As shown in Figure 6, the complete assembly and testing of the Original Entry Point based design is done once the components are assembled.

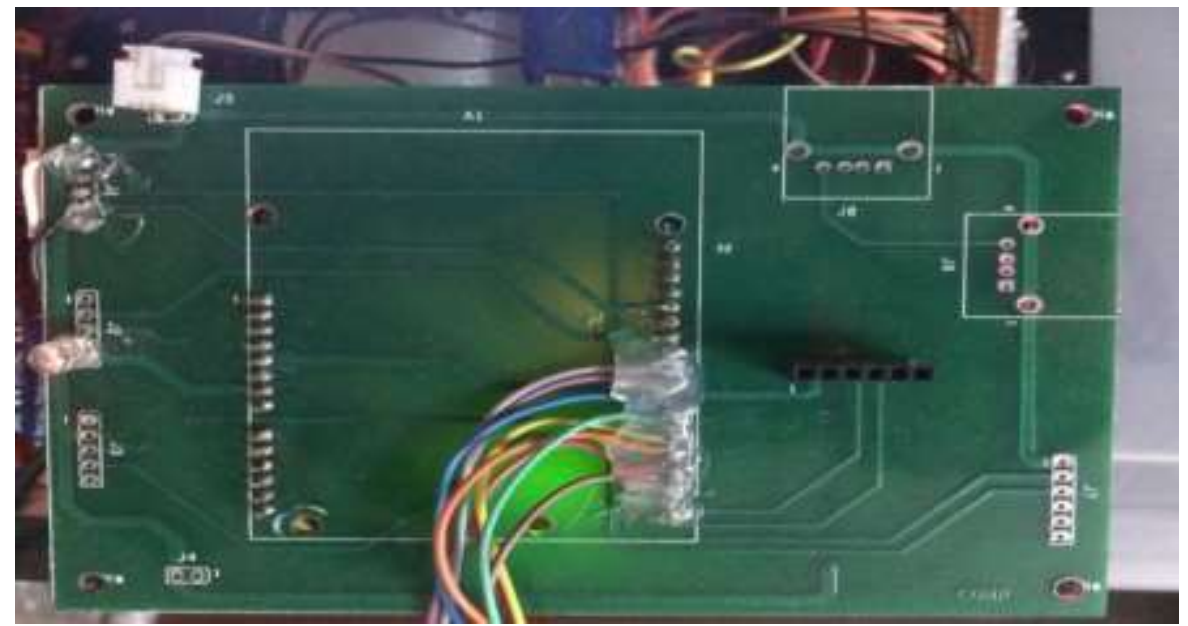

Figure 6 Final Components Assembly (OEP Design)

\section{STANDALONE CONTROLLER CARD DESIGN (TYPE B: DUAL ENTRY DESIGN)}

The Dual Entry design refers to the Hardware design which incorporates the use of two entry points for the dumping of the firmware. An additional entry point is an extended design to the same Rx and Tx (Serial pins) of the controller to a USB to Serial converter IC such as FT232. This can be used for designing a proprietary controller card with preset fuse and lock bits. The VID (Vendor Id) and PID (Product Id) can also be set while burning the Bootloader onto the controller. Furthermore, the Original Entry 
International Journal of Advances in Scientific Research and Engineering (ijasre), Vol 5 (6), June-2019

Point can still be used for Serial communication if the required components are populated accordingly. Either the Additional point or the Original Point of Entry facilitates Serial Communication to the controller[1].

Table 2 Design Considerations and Complexity of Dual Entry Design based Hardware Design

\begin{tabular}{|l|l|}
\hline DESIGN CONSIDERATION & COMPLEXITY \\
\hline Schematic Design & Complex \\
\hline Bill of Materials & Increased \\
\hline Component Placement & Complex \\
\hline Routing Considerations & High \\
\hline Layout Visualization & Complex \\
\hline Post Fabrication/Testing & Simple \\
\hline
\end{tabular}

\subsection{Schematic Design (DE Design)}

The Dual Entry Design uses a complete complex Schematic design which builds the complete circuit using a bottom-up approach and also includes the Controller design. Furthermore, an Additional Point of entry is provided alongside the Original Point[5].

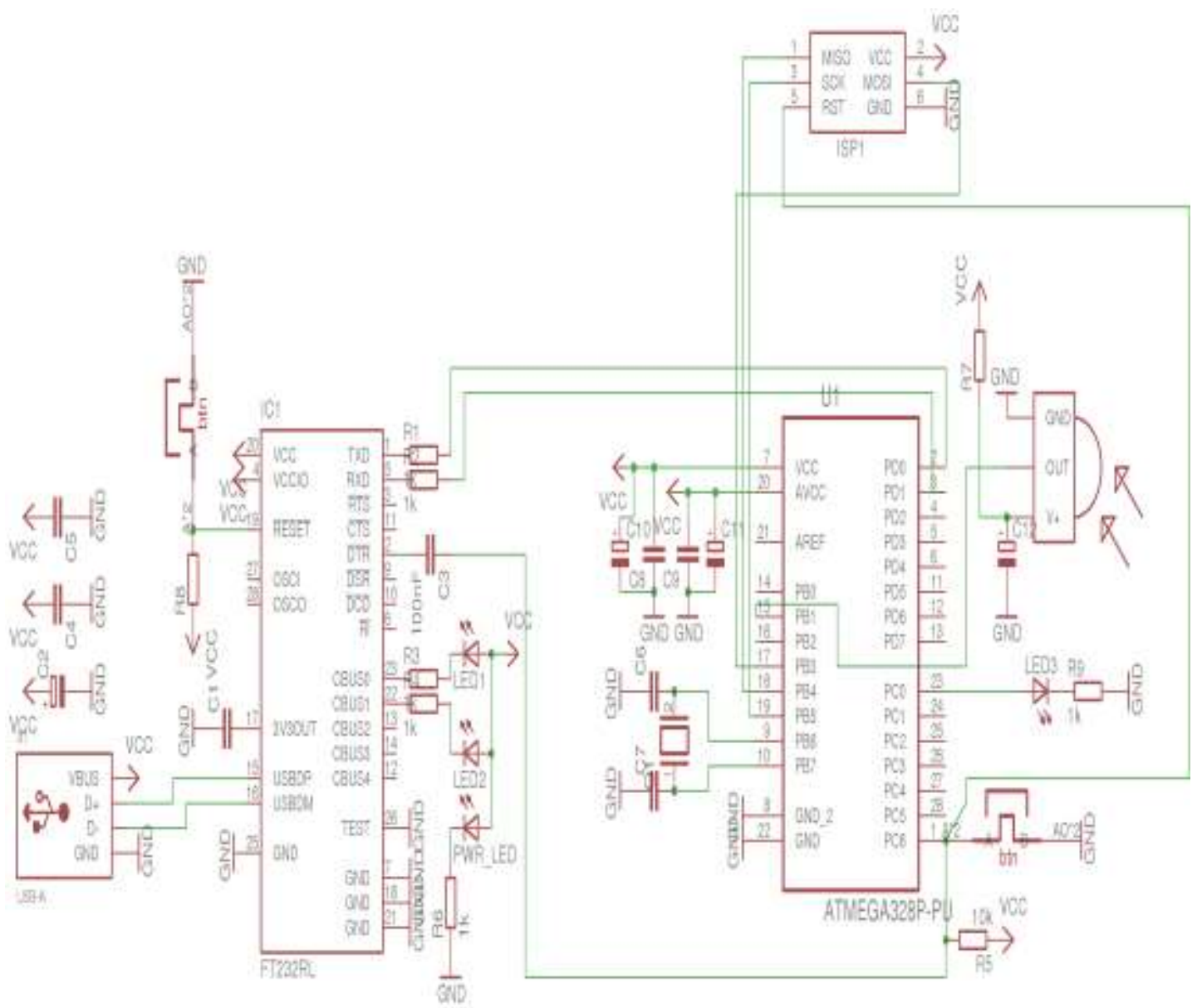

Figure 7 PCB Schematic using an FTDI chip and ATMega328 controller for DE Design

\subsection{Board Layout (DE Design)}

The component Placement with the board layout illustrated in Figure 8 shows an extended routing requirement of the Dual Entry Design. The peripherals such as Power Ports, Wireless Connectivity (Bluetooth), Reset Port, Digital and Analog O/P ports are included in the controller Schematic as well. Standard design considerations are the same as that of the OEP Technique with the Power supply and Ground tracks using $0.4 \mathrm{~mm}$ track width for undeterred power transmission with EMI Shielding and reduced Power losses and the Digital signals are designed with a standard track width of $0.24 \mathrm{~mm}[1]$. 
International Journal of Advances in Scientific Research and Engineering (ijasre), Vol 5 (6), June-2019

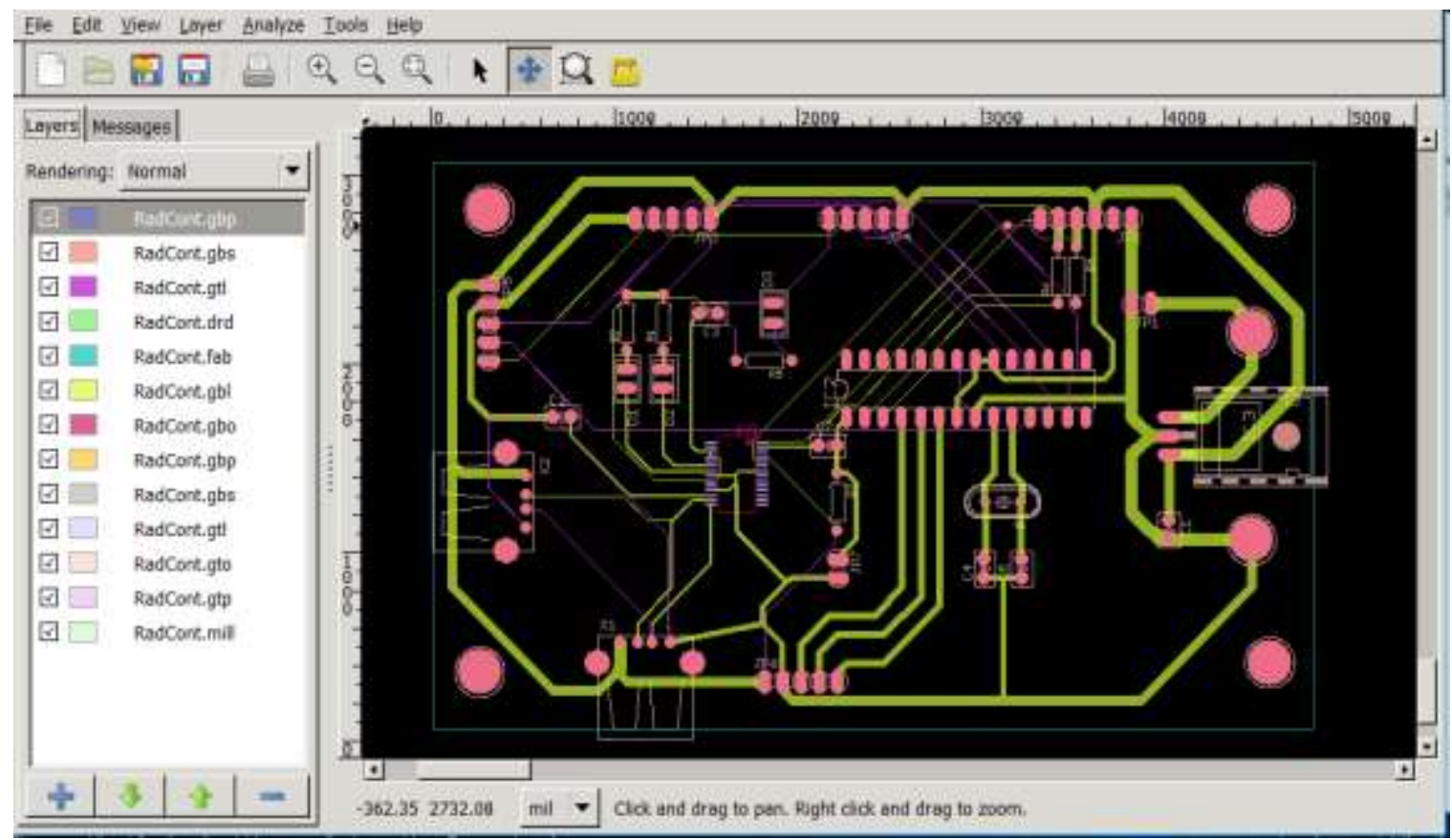

Figure 8 Board Layout with TOP LAYER=YELLOW, BOTTOM LAYER=PURPLE

\subsection{FTDI Connectivity Simulated Circuitry}

As seen in Figure 9, the Dual Entry based design is simulated with an additional FTDI (FT232 IC) used to upload the firmware to the controller used (ATMega328). The entire design of the controller schematic is included with the peripheral schematic[2].

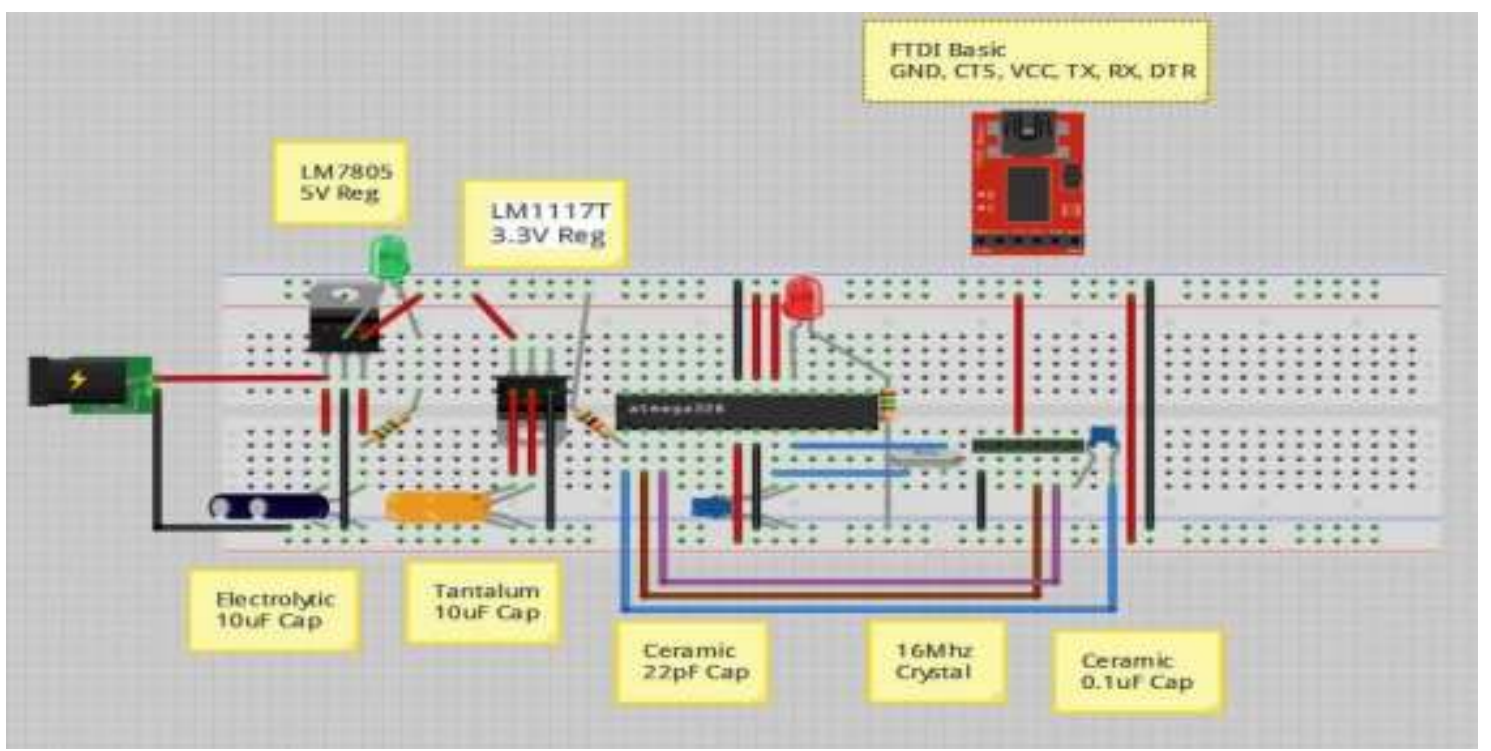

Figure 91 FTDI Connection Schematic

\subsection{Post Fabrication (DE Design)}

The Dual Entry Design PCB shown in Figure 10 shows the final components assembly of the PCB with OEP (Original Entry Point) based design used alongside the additional DE Design. The following image shows the Top and Bottom Layers post Fabrication[2]. 

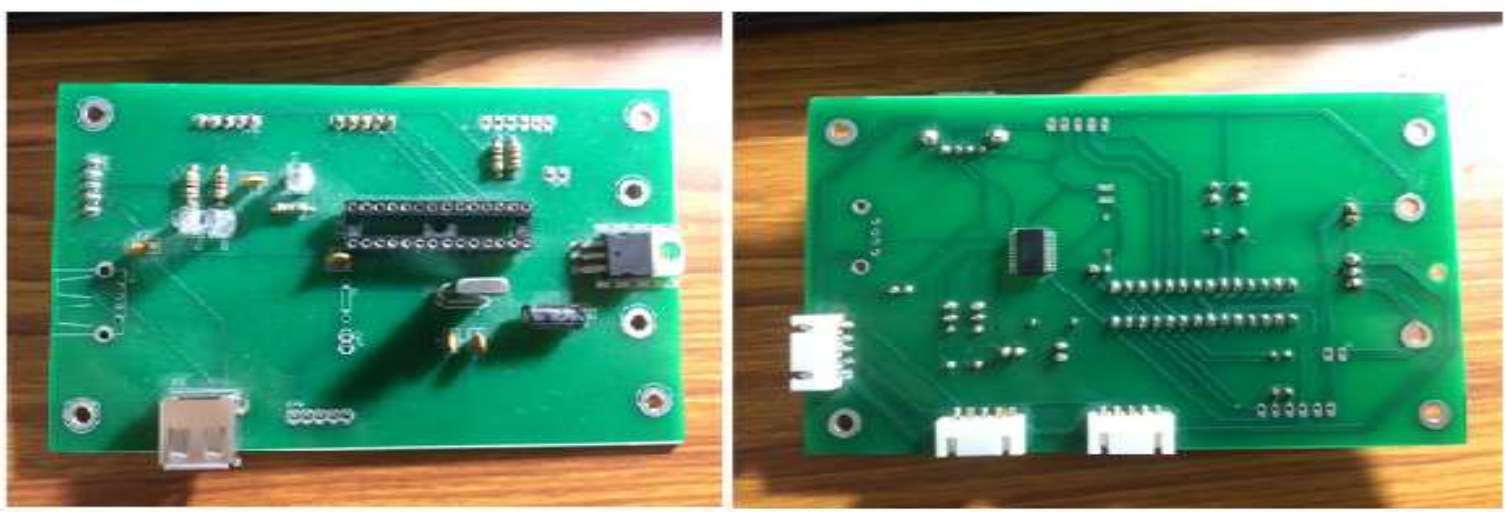

Figure 10 PCB Fabrication Top and Bottom Layers of the DE Design Post Fabrication

\section{RESULTS AND DISCUSSION}

The final results of both the techniques (OEP and DE) propose a method by which secure firmware upload can take place to a proprietary controller card. The main difference between the two techniques is the complexity of the design of the second technique (DE Design). The extended functionality of setting fuse and lock bits account for the increase in complexity with an additional feature of using either port for Serial Communication. The following table provides a comparison between the two techniques with respect to ease of design and real-time considerations.

Table 3 Comparision between OEP and DE Design

\begin{tabular}{|l|l|l|}
\hline FACTOR & OEP DESIGN & DE DESIGN \\
\hline Schematic Complexity & Less Complex & High Complexity \\
\hline Routing Complexity & Lesser tracks & Complex Tracks \\
\hline Board Layout & Less Density & High Density \\
\hline Post Fabrication Testing & Simple & Complex \\
\hline Cost & Cheap & Expensive \\
\hline CNC Complexity & Less & High \\
\hline Drill Complexity & Less & High \\
\hline
\end{tabular}

\section{CONCLUSION}

The holistic design of both techniques is discussed and compared in detail. The fabrication considerations of the design highly depend on the required output. Gerber files were generated for both the PCB's and the output is described above. Post Fabrication and Testing techniques vary and depend on the specific design. Many such standalone controller cards using OEP and DE Design techniques can be designed and fabricated to mount generic controllers of different families such as Atmel, Microchip (PIC), Texas Instrument and NXP[3].

\section{REFERENCES}

1. Agah, A., and C.T. Pierik. "Design and fabrication of a team of robots in hardware." Proceedings of the 1996 IEEE IECON. 22nd International Conference on Industrial Electronics, Control, and Instrumentation. IEEE, 1996. 1577 - 1582.

2. Ma, Wenheng, Ningmei Yu, Ga Liu, Qilong Sheng, Meng Wang, and Li Dai. "A Pre-Fabrication Malicious Hardware Implementation Method." 2018 14th IEEE International Conference on Solid-State and Integrated Circuit Technology (ICSICT). IEEE, 2018. 1-3. 
International Journal of Advances in Scientific Research and Engineering (ijasre), Vol 5 (6), June-2019

3. Ou, Jack, Alberto Maldonado, Chio Saephan, Farid Farahmand, and Michael Caggiano. "A low-cost PCB fabrication process." 2014 IEEE 64th Electronic Components and Technology Conference (ECTC). IEEE, 2014. 2159 - 2162.

4. Ramani, K., and R.L. Haggard. "A survey of techniques used in the synthesis of hardware from $\mathrm{C} / \mathrm{C}++$ as a part of hardware/software co-design." Proceedings of the 33rd Southeastern Symposium on System Theory (Cat. No.01EX460). IEEE, 2001. $301-304$.

5. Shinde, Shrihari, and H. Muthurajan. "PCB fabrication unit for electronics circuit prototyping." 2016 IEEE International Conference on Advances in Electronics, Communication and Computer Technology (ICAECCT). IEEE, 2016. 1-5. 